\title{
Spectrum Utilization Maximization in Energy Limited Cooperative Cognitive Radio Networks
}

\author{
Yan Long ${ }^{\dagger}$, Hongyan $\mathrm{Li}^{\dagger *}$, Hao Yue ${ }^{\ddagger}$, Miao Pan ${ }^{\S}$ and Yuguang Fang ${ }^{\ddagger}$ \\ ${ }^{\dagger}$ State Key Laboratory of Integrated Services Networks, Xidian University, Xi'an, China \\ $\ddagger$ Department of Electrical and Computer Engineering, University of Florida, Gainesville, FL \\ $\S$ Department of Computer Science, Texas Southern University, TX
}

\begin{abstract}
In cooperative cognitive radio networks (CCRNs), through cooperating with primary transmissions, secondary users (SUs) could access the spectrum resource when primary users (PUs) are transmitting. The existing schemes in CCRNs allocate the spectrum resource only to the cooperative relay SU. However, this may lead to the waste of spectrum resource, especially when the relay SU has light traffic load or poor channel condition. To better utilize the spectrum among all SUs in a secondary network, we design a spectrum resource utilization maximization scheme with joint consideration of relay selection and spectrum scheduling problems. With the goal to maximize the throughput of the secondary network, our scheme allocates spectrum among all SUs according to the diversity of secondary traffic load and the channel conditions. Besides, considering that the SUs are always energy limited, we also formulate the energy constraint for each $\mathrm{SU}$ to avoid the energy consumption exceeding the total available energy. Moreover, we study the resource allocation problem from long-term view under dynamic network setting, and design an online algorithm to solve it. Through extensive simulations, we show that the proposed scheme outperforms the existing schemes in terms of secondary network throughput.
\end{abstract}

\section{INTRODUCTION}

Recently, the boost of wireless services leads to a dramatic increase in the demand for spectrum resource. However, the current fixed spectrum allocation of Federal Communications Commission (FCC) can not utilize spectrum resource efficiently. One of the promising approaches to increase spectrum efficiency is cognitive radio technology, which enables secondary users (SUs) to sense spectrum and opportunistically access idle spectrum bands owned by primary users (PUs) [1], [2].

Cooperative cognitive radio network (CCRN) [3], [4] is a new paradigm in cognitive radio networks. Different from traditional cognitive radio networks in [5], [6], by integrating cooperative communications, SUs in a CCRN could still gain transmission opportunities even when PUs are active to transmit. To further illustrate CCRNs, consider a toy example in Fig. 1. $P U_{1}$ is transmitting to $P U_{2}$, and in the same geographic area, $S U_{i}$ and $S U_{j}$ are intending to send data to secondary access point (SAP). We consider that with $S U_{i}$ as the cooperative relay, the relay link $P U_{1}-S U_{i}-P U_{2}$ is superior to the direct-transmission link $P U_{1}-P U_{2}$ in terms of primary transmission rate [7]. Then, $S U_{i}$ can be involved to increase the primary transmission rate and help the primary

*Corresponding author (email: hyli@xidian.edu.cn)

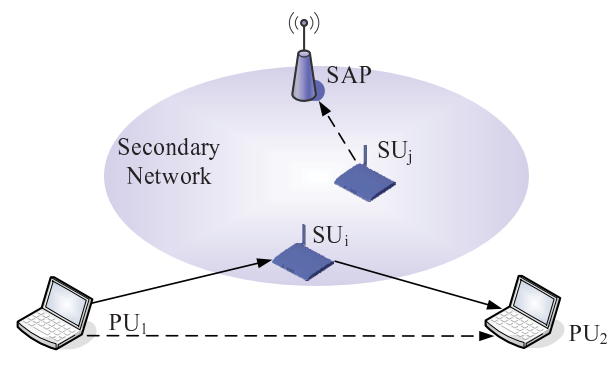

Fig. 1. An illustrative example of CCRNs

transmission. Since the primary transmission is finished earlier with the cooperation, more vacant time period is created. In return, this vacant period from the cooperation can be further used by SUs for their own secondary transmissions. In this way, CCRNs bring a win-win situation to both PUs and SUs.

Recently, the cross-layer resource allocation problem in CCRNs has been studied in [8]-[10]. Simeone et al. in [8] first propose the resource allocation problem under time invariant CCRNs, and formulate a Stackelberg game to obtain the optimal relay selection and secondary transmission scheduling. Zhang et al. in [9] study a similar problem, but consider a revenue issue in the utility function of PUs. Khalil et al. in [11] extend the resource allocation problem into dynamic CCRNs, where the network setting such as channel fading and traffic arrival rate changes over time. $\mathrm{Xu}$ and $\mathrm{Li}$ in [10] investigate the problem in multi-channel CCRNs. They consider OFDMA based multi-channel primary networks, and add the channel diversity into resource allocation problem.

An implicit premise of these works is that only the relay $\mathrm{SU}$ (e.g., $S U_{i}$ in Fig. 1) could use vacant time period which is created from cooperation. However, if we maximize the throughput of the secondary network, this premise may result in waste of spectrum resource especially when the following cases apply: i) the relay SU does not have secondary data to transmit, while other SUs (e.g., $S U_{j}$ ) have a large amount of data to transmit; ii) the channel condition from other SUs to $\mathrm{SAP}$ is much better than that of the relay SU. Therefore, to maximize the secondary network throughput and better utilize the spectrum resource derived from cooperation, we should optimally allocate such resource among all SUs based on their channel condition and traffic amount, rather than allowing 
only the relay $\mathrm{SU}$ to access $\mathrm{it}^{1}$. More specifically, like the infrastructure secondary service provider (SSP) in [12], the SAP could be assumed to conduct the centralized control and spectrum resource collaboration among all SUs to improve the secondary network performance.

Besides, given the fact that SUs are always energy limited, a SU may not be willing to spend too much energy for cooperation, since it needs to maintain energy for its own secondary transmissions. Both cooperation energy and secondary transmission energy consumption are challenging the energy limitation. Thus, when we study the spectrum allocation problem in CCRNs, the relay selection and secondary transmission scheduling should be considered jointly. Even though [13] has studied a similar energy issue in CCRNs, it mainly focuses on single SU scenario and fails to consider channel diversity when conducting resource allocation in CCRNs.

From the observations above, we design a novel crosslayer resource allocation scheme in CCRNs, to maximize spectrum utilization among all SUs in a secondary network. We consider the relay selection and secondary transmission scheduling jointly, with the objective to maximize the throughput of the secondary network. The relay selection chooses the optimal SU to cooperate with primary communications, and the secondary transmission scheduling optimally allocates spectrum resource among all secondary transmissions. Moreover, we specify an energy constraint for each SU to find the trade-off between these two aspects. In addition, since the network setting and primary activity is time varying in practical CCRNs, we study the average performance over a relatively long period of time to integrate the dynamic feature. Finally, we design an optimal online algorithm, which solves the long-term optimization problem by only requiring instant network information. Simulations demonstrate that the proposed scheme has great advantages over the previous schemes in terms of secondary network throughput in CCRNs.

The rest of this paper is organized as follows. In Section II, we introduce the system model. In Section III, we formulate the resource allocation problem in CCRNs with the goal of spectrum utilization maximization. We propose an optimal online algorithm to solve it in Section IV, and present the numerical simulation results in Section V. Finally, we conclude this paper in Section VI.

\section{SySTEM ModeL}

We consider a CCRN as shown in Fig. 2. There is a primary network with primary node set $N_{P}$ and link set $L_{P}$, and a secondary network with secondary node set $N_{S}$ and one SAP. Each SU has data to send to the SAP. In specific applications, the SAP can be viewed as the SSP in the architecture [12], the femtocell base station in [13] or the sink node in sensor networks [14]. We use capital letters to denote PUs, small letters to denote SUs and 0 to denote SAP. Also, we denote a link by its transmitter-receiver pair. For example, $M N$

\footnotetext{
${ }^{1}$ Here, we assume that to maximize the secondary network throughput, the relay SU agrees to share this vacant period with other SUs in the secondary network even though they don't contribute to this cooperative transmission.
}

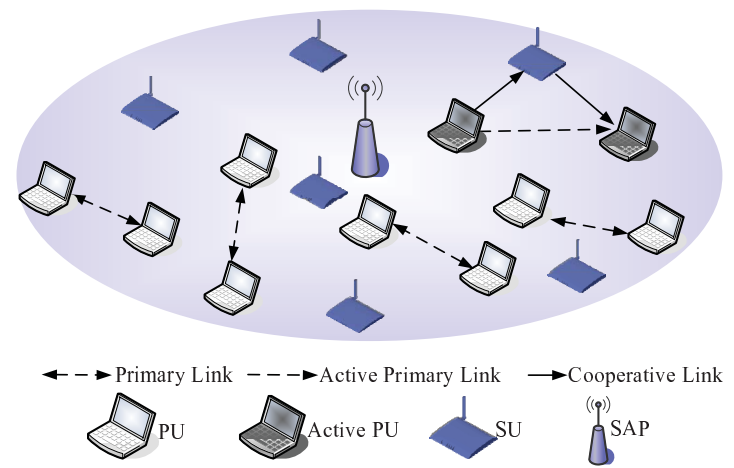

Fig. 2. Toy topology of a CCRN

\begin{tabular}{|c|c|c|c|c|c|}
\hline$\alpha_{\mathrm{Mi}(\mathrm{t})}$ & $\beta_{\mathrm{iN}(\mathrm{t})}$ & $\gamma_{10(t)} \mid$ & $\gamma_{20(t)}$ & $\gamma_{30(t)}$ & $\ldots$ \\
\hline \multicolumn{2}{|c|}{$\overrightarrow{\text { Cooperation Period }}$} & \multicolumn{4}{|c|}{ Secondary Transmission Period } \\
\hline
\end{tabular}

Fig. 3. Time splitting in a control interval

represents the primary link from $M$ to $N, i N$ means the link from SU $i$ to PU $N$, and $i 0$ is the link from SU $i$ to the SAP. Time is discretized into control intervals. In each interval, similar to SSP in [12], SAP is assumed to conduct centralized control through the information exchange with users in the CCRN over a common control channel. Note that PUs and SUs are willing to exchange information with SAP since both of them could benefit from SAP's control.

In interval $t$, we have $\eta_{M N}(t)=1$ if the primary link $M-N$ is active; otherwise, $\eta_{M N}(t)=0$. We consider a small geographic area where all SUs in the secondary network can sense the same spectrum environment. In this small area, primary links may interfere with each other. To avoid such interference among PUs, we assume that at most one primary link could be activated in each interval, i.e., $\sum_{M N \in L_{P}} \eta_{M N}(t) \leq 1$.

In interval $t$, if primary link $M-N$ is activated and SU $i$ cooperates as a relay, interval $t$ will be split into cooperation period and secondary transmission period, as shown in Fig. 3. During the cooperation period, $M$ first transmits data to $N$ in period $\alpha_{M i}(t)$, and meanwhile, $\mathrm{SU} i$ overhears the data due to the broadcast nature of wireless transmission. Then, $i$ forwards the received data to $N$ in period $\beta_{i N}(t)$. According to the secondary transmission scheduling strategy, the secondary transmission period is further divided into $\left|N_{S}\right|$ sub-periods, where $\gamma_{i 0}(t)$ represents the time allocated to $i$ to conduct its own secondary transmission. We assume channel fading are independent and identically distributed (i.i.d.) across intervals, and independent across different users. Channel gains change over time, but remain constant within each interval, implying channels and nodes in the CCRN are relatively stationary, which is consistent with practical applications for wireless LANs. This also hints that the time interval will not be too short (e.g., the length of the interval could be tens of milliseconds for a slow fading channel [15]), thus the time splitting during the interval is meaningful. 
In interval $t$, for SU $i$, we use $\lambda_{i}(t)$ to denote the traffic arrival rate at its transport layer, and $A_{i}(t)$ to denote the admitted rate from the transport layer to the network layer. $A_{i}(t)$ is usually bounded by a positive constant $A_{\max }$ to avoid infinite data to the network layer. Besides, $h_{M N}(t)$ represents the instant channel gain of link $M-N$ in interval $t$. Similarly, we define $h_{M i}(t), h_{i N}(t)$ and $h_{i 0}(t)$ for links $M-i, i-N$ and $i$-0, respectively. We consider fixed transmission power for all users in the CCRN. Let $P$ and $P^{\prime}$ represent the primary and secondary transmission power, respectively. Without loss of generality, we assume the length of a control interval is 1 , the spectrum bandwidth is 1 , and the noise power is $N_{0}$.

\section{PROBLEM Formulation}

\section{A. Relay Selection Constraint}

For simplicity, we assume that at most one SU is selected as the relay in each time interval, i.e.,

$$
\eta_{M N}(t) \sum_{i \in N_{S}} \theta_{M N}^{i}(t) \leq 1,
$$

where $\theta_{M N}^{i}(t)=1$ means that the $\mathrm{SU} i$ is selected as the relay for primary link $M-N$; otherwise, we set $\theta_{M N}^{i}(t)=0$.

\section{B. Cooperation Constraints for SUs and PUs}

We assume the Decode-and-Forward mode [7] is adopted at the relay node. Thus, the transmission rate over relay link $M-i-N$ is,

$$
\begin{array}{r}
R_{M N}^{i}(t)=\min \left\{\theta_{M N}^{i}(t) \eta_{M N}(t) \alpha_{M i}(t) \log \left(1+\frac{\left|h_{M i}(t)\right|^{2} P}{N_{0}}\right),\right. \\
\left.\theta_{M N}^{i}(t) \eta_{M N}(t) \beta_{i N}(t) \log \left(1+\frac{\left|h_{i N}(t)\right|^{2} P^{\prime}}{N_{0}}\right)\right\} .
\end{array}
$$

With cooperative communications, all the data received by the relay SU $i$ from PU $M$ in period $\alpha_{M i}(t)$, must be delivered from $i$ to PU $N$ in period $\beta_{i N}(t)$. Therefore, we have the following cooperation constraint for SUs:

$$
\begin{gathered}
\theta_{M N}^{i}(t) \eta_{M N}(t) \alpha_{M i}(t) \log \left(1+\frac{\left|h_{M i}(t)\right|^{2} P}{N_{0}}\right) \leq \\
\theta_{M N}^{i}(t) \eta_{M N}(t) \beta_{i N}(t) \log \left(1+\frac{\left|h_{i N}(t)\right|^{2} P^{\prime}}{N_{0}}\right) .
\end{gathered}
$$

When (2) holds, $R_{M N}^{i}(t)$ could be rewritten as $R_{M N}^{i}(t)=$ $\theta_{M N}^{i}(t) \eta_{M N}(t) \alpha_{M i}(t) \log \left(1+\frac{\left|h_{M i}(t)\right|^{2} P}{N_{0}}\right)$.

For PUs, the primary transmission rate under cooperation mode should be no less than that without cooperation, so that they have incentive to cooperate with SUs. Therefore, we have the cooperation constraint for PUs as below,

$$
\begin{aligned}
& \eta_{M N}(t) \log \left(1+\frac{\left|h_{M N}(t)\right|^{2} P}{N_{0}}\right) \leq \\
& \sum_{i \in N_{S}} \theta_{M N}^{i}(t) \eta_{M N}(t) \alpha_{M i}(t) \log \left(1+\frac{\left|h_{M i}(t)\right|^{2} P}{N_{0}}\right)+ \\
& \quad\left(1-\sum_{i \in N_{S}} \theta_{M N}^{i}(t)\right) \eta_{M N}(t) \log \left(1+\frac{\left|h_{M N}(t)\right|^{2} P}{N_{0}}\right),
\end{aligned}
$$

where the left-hand-side (LHS) represents the primary transmission rate over direct link $M-N$. The right-hand-side (RHS) integrates the rate into two parts: if the primary activity is cooperated with a SU, i.e., $\sum_{i \in N_{S}} \theta_{M N}^{i}(t)=1$, the RHS equals to $R_{M N}^{i}(t)$; while if it transmits under non-cooperation mode, i.e., $1-\sum_{i \in N_{S}} \theta_{M N}^{i}(t)=1$, the RHS equals to LHS.

\section{Secondary Transmission Constraint}

Recall that the length of a control interval is 1, the summation of all sub-periods should satisfy

$$
\begin{gathered}
\sum_{M N \in L_{P}} \eta_{M N}(t)\left[\sum_{i \in N_{S}} \theta_{M N}^{i}(t)\left(\alpha_{M i}(t)+\beta_{i N}(t)\right)+\right. \\
\left.\left(1-\sum_{i \in N_{S}} \theta_{M N}^{i}(t)\right)\right]+\sum_{i \in N_{S}} \gamma_{i 0}(t) \leq 1 .
\end{gathered}
$$

In constraint (4), when all primary links are inactive, i.e., $\sum_{M N \in L_{P}} \eta_{M N}(t)=0, \sum_{i \in N_{S}} \gamma_{i 0}(t) \leq 1$ holds. This means the interval could be totally used for secondary transmissions. However, in the case where a primary link is activated and no SU is selected as the relay, constraint (4) becomes $1+\sum_{i \in N_{S}} \gamma_{i 0}(t) \leq 1$. In this case, SUs can not access this interval to conduct transmissions. Moreover, if a primary link is activated and a SU is selected as the relay, we have $\alpha_{M i}(t)+\beta_{i N}(t)+\sum_{i \in N_{S}} \gamma_{i 0}(t) \leq 1$, which constrains the summation of sub-periods $\alpha_{M i}(t), \beta_{i N}(t)$ and $\gamma_{i 0}(t)$ will not exceed 1 .

\section{Secondary Queueing Stability Constraint}

Each SU $i$ maintains a queue $Q_{T_{i}}(t)$ on its transport layer and a queue $Q_{N_{i}}(t)$ on the network layer. The queue update equations are

$$
\begin{aligned}
Q_{T_{i}}(t+1)= & \max \left\{Q_{T_{i}}(t)-A_{i}(t), 0\right\}+\lambda_{i}(t), \\
Q_{N_{i}}(t+1)= & \max \left\{Q_{N_{i}}(t)-\gamma_{i 0}(t) \log \left(1+\frac{\left|h_{i 0}(t)\right|^{2} P^{\prime}}{N_{0}}\right), 0\right\} \\
& +A_{i}(t),
\end{aligned}
$$

where $\gamma_{i 0}(t) \log \left(1+\frac{\left|h_{i 0}(t)\right|^{2} P^{\prime}}{N_{0}}\right)$ is the instant secondary transmission rate calculated based on secondary transmission scheduling.

Our objective is to maximize the secondary network throughput, while keeping the network layer queue $Q_{N_{i}}(t)$ stable on each SU. To this end, for $Q_{N_{i}}(t)$, its long-term averaged input rate (i.e., admitted rate) must be less than its long-term averaged output rate (i.e., transmission rate) [16], [17]. That is,

$$
\begin{aligned}
& \lim _{T \rightarrow \infty} \frac{1}{T} \sum_{t=0}^{T-1} \mathbb{E}\left[A_{i}(t)\right]< \\
& \lim _{T \rightarrow \infty} \frac{1}{T} \sum_{t=0}^{T-1} \mathbb{E}\left[\gamma_{i 0}(t) \log \left(1+\frac{\left|h_{i 0}(t)\right|^{2} P^{\prime}}{N_{0}}\right)\right] .
\end{aligned}
$$

In addition, note that $A_{i}(t)$ is bounded by the transport layer queue $Q_{T_{i}}(t)$ as well as $A_{\max }$. We have

$$
A_{i}(t) \leq \min \left\{A_{\max }, Q_{T_{i}}(t)\right\} .
$$




\section{E. Energy Constraint for SUs}

To avoid the energy consumption exceeding the total available energy, we introduce $E_{\text {ave }}^{i}$ to denote the averaged available energy of $i$ during a control interval. For each SU, $E_{\text {ave }}^{i}$ is pre-determined and is proportional to its total available energy amount. In interval $t$, $i$ 's energy consumption consists of two parts: the cooperation energy $\sum_{M N \in L_{P}} \eta_{M N}(t) \theta_{M N}^{i}(t) \beta_{i N}(t) P^{\prime}$ and the secondary transmission energy $\gamma_{i 0}(t) P^{\prime}$. For each SU, we design the energy constraint to specify that its long-term averaged energy consumption is less than the $E_{\text {ave }}^{i}$, which is expressed as,

$$
\begin{aligned}
& \lim _{T \rightarrow \infty} \frac{1}{T} \sum_{t=0}^{T-1} \mathbb{E}\left[\sum_{M N \in L_{P}} \eta_{M N}(t) \theta_{M N}^{i}(t) \beta_{i N}(t) P^{\prime}+\gamma_{i 0}(t) P^{\prime}\right] \\
& \quad<E_{\text {ave }}^{i}
\end{aligned}
$$

In order to satisfy constraint (7), we introduce a virtual energy queue $Q_{E_{i}}(t)$ for each SU [18]. The update equation of the virtual queue is

$$
\begin{aligned}
Q_{E_{i}}(t+1)= & \max \left\{Q_{E_{i}}(t)-E_{\text {ave }}^{i}, 0\right\}+ \\
& \sum_{M N \in L_{P}} \eta_{M N}(t) \theta_{M N}^{i}(t) \beta_{i N}(t) P^{\prime}+\gamma_{i 0}(t) P^{\prime} .
\end{aligned}
$$

Similar to the network layer queue $Q_{N_{i}}(t)$, if $Q_{E_{i}}(t)$ is stable, its input rate will be less than its output rate [17]. In that case, the energy constraint (7) holds.

\section{F. Objective Function}

The objective is to maximize the secondary network throughput, which is defined as the summation of long-term averaged admitted rate over all SUs:

$$
\max \sum_{i \in N_{S}} \lim _{T \rightarrow \infty} \frac{1}{T} \sum_{t=0}^{T-1} \mathbb{E}\left[A_{i}(t)\right] .
$$

\section{G. Overall Optimization Problem}

Based on the analysis above, to maximize the spectrum utilization among all SUs with energy limitation, we jointly formulate the relay selection and secondary transmission scheduling problems with the goal of secondary network throughput maximization as follows:

$$
\max \sum_{i \in N_{S}} \lim _{T \rightarrow \infty} \frac{1}{T} \sum_{t=0}^{T-1} \mathbb{E}\left[A_{i}(t)\right]
$$

subject to:

$$
\begin{array}{ll}
(1)-(7) & \\
\theta_{M N}^{i}(t) \in\{0,1\}, & \forall i \in N_{S}, \forall M N \in L_{P}, \forall t \\
0 \leq \alpha_{M i}(t) \leq 1, & \forall i \in N_{S}, \forall M N \in L_{P}, \forall t \\
0 \leq \beta_{i N}(t) \leq 1, & \forall i \in N_{S}, \forall M N \in L_{P}, \forall t \\
0 \leq \gamma_{i 0}(t) \leq 1, & \forall i \in N_{S}, \forall t,
\end{array}
$$

where $A_{i}(t), \theta_{M N}^{i}(t), \alpha_{M i}(t), \beta_{i N}(t)$ and $\gamma_{i 0}(t)$ are decision variables, and $P, P^{\prime}, A_{\max }, E_{\text {ave }}^{i}, N_{0}, Q_{T_{i}}(t), \eta_{M N}(t)$, $h_{M N}(t), h_{M i}(t), h_{i N}(t)$ and $h_{i 0}(t)$ are constants.
In problem (8), the long-term performance is considered in both the objective function and constraints (5) and (7), where the statistical network information, e.g., channel conditions, traffic arrival rate and primary activities, is required. However, the statistics is hard to obtain in practical CCRNs, and this does pose significant challenges to solution process. Therefore, we develop an optimal online algorithm in Section IV. In the algorithm, the instant optimization problems of each control interval are defined, and the long-term optimum can be achieved through running the algorithm over a period of time. In each interval, the instant optimization problem is solved based on the instant information of the current interval.

\section{An Optimal Online Algorithm}

To solve the long-term optimization problem (8), we design an optimal online algorithm according to Lyapunov optimization tool [16]. Its basic idea is to minimize the Lyapunov driftplus-penalty function [17], which is expressed as

$$
\min \triangle L(t)-V \sum_{i \in N_{S}} \mathbb{E}\left[A_{i}(t) \mid Q_{N_{i}}(t), Q_{E_{i}}(t)\right],
$$

where $\triangle L(t)=\frac{1}{2} \mathbb{E}\left[\sum_{i \in N_{S}} Q_{N_{i}}^{2}(t+1)+Q_{E_{i}}^{2}(t+1)-Q_{N_{i}}^{2}(t)-\right.$ $\left.Q_{E_{i}}^{2}(t) \mid Q_{N_{i}}(t), Q_{E_{i}}(t)\right]$ is the Lyapunov drift function of problem (8), and $V \geq 0$ is a pre-defined constant to balance the tradeoff between the network throughput and network delay [19]. Specifically, from Lyapunov drift analysis [16], [17], with the increase of $V$, the objective of the proposed online algorithm is close to the optimal throughput. However, the network delay is also increasing.

Moreover, the minimization of the Lyapunov drift-pluspenalty function can be further decomposed into instant admitted control and network control problems in each interval. The details is shown as follows.

\section{A. Admitted Rate Control at SUs}

In interval $t$, each $\mathrm{SU} i$ locally solves the following optimization problem in terms of its admitted rate $A_{i}(t)$.

$$
\begin{aligned}
& \min A_{i}(t)\left(Q_{N_{i}}(t)-V\right) \\
& \text { subject to: } \\
& A_{i}(t) \leq \min \left\{A_{\max }, Q_{T_{i}}(t)\right\}, \quad \forall i \in N_{S}, \forall t .
\end{aligned}
$$

\section{B. Centralized Control at SAP}

In interval $t$, the SAP first collects the current network setting information from PUs and SUs. Then, it solves the optimization formulation (9) below to attain the optimal relay selection and secondary transmission scheduling strategy for the current interval.

$$
\begin{aligned}
& \min \sum_{i \in N_{S}} Q_{E_{i}}(t)\left(\sum_{M N \in L_{P}} \eta_{M N}(t) \theta_{M N}^{i}(t) \beta_{i N}(t) P^{\prime}\right. \\
& \left.\quad+\gamma_{i 0}(t) P^{\prime}\right)-\sum_{i \in N_{S}} Q_{N_{i}}(t) \gamma_{i 0}(t) \log \left(1+\frac{\left|h_{i 0}(t)\right|^{2} P^{\prime}}{N_{0}}\right)
\end{aligned}
$$

subject to: 


$$
\begin{array}{ll}
(1)-(4), & \\
\theta_{M N}^{i}(t) \in\{0,1\}, & \forall i \in N_{S}, \forall M N \in L_{P}, \forall t \\
0 \leq \alpha_{M i}(t) \leq 1, & \forall i \in N_{S}, \forall M N \in L_{P}, \forall t \\
0 \leq \beta_{i N}(t) \leq 1, & \forall i \in N_{S}, \forall M N \in L_{P}, \forall t \\
0 \leq \gamma_{i 0}(t) \leq 1, & \forall i \in N_{S}, \forall t .
\end{array}
$$

Note that since the variable $\theta_{M N}^{i}(t)$ is binary, (9) becomes a mixed-integer non-linear programming problem, which is hard to solve. Fortunately, we observe that when problem (9) under non-cooperation mode, i.e., $\left\{\theta_{M N}^{i}(t)=0, i \in N_{S}, M N \in\right.$ $\left.L_{P}\right\}$, it becomes a linear programming problem, which is easy to solve. Similarly, for problem (9) under the cooperation mode, for each $\mathrm{SU} i$, if we postulate that this $\mathrm{SU}$ is the cooperative relay, i.e., $\left\{\theta_{M N}^{i}(t)=1, M N \in L_{P}\right\}$ and $\left\{\theta_{M N}^{i^{\prime}}(t)=0, i^{\prime} \neq i, i^{\prime} \in N_{S}, M N \in L_{P}\right\}$, then (9) also converts to a linear programming problem. For this reason, problem (9) can be solved through the following steps. First, SAP fixes $\theta_{M N}^{i}(t)$ and solves $\left|N_{S}\right|+1$ linear programming problems under non-cooperation and cooperation modes, respectively. After that, SAP compares the $\left|N_{S}\right|+1$ objective values and chooses the minimum one as the optimum. In this way, the optimal $\theta_{M N}^{i}(t), \alpha_{M i}(t), \beta_{i N}(t)$ and $\gamma_{i 0}(t)$ are obtained and problem (9) is easily solved over a finite set. The details is shown in Algorithm 1.

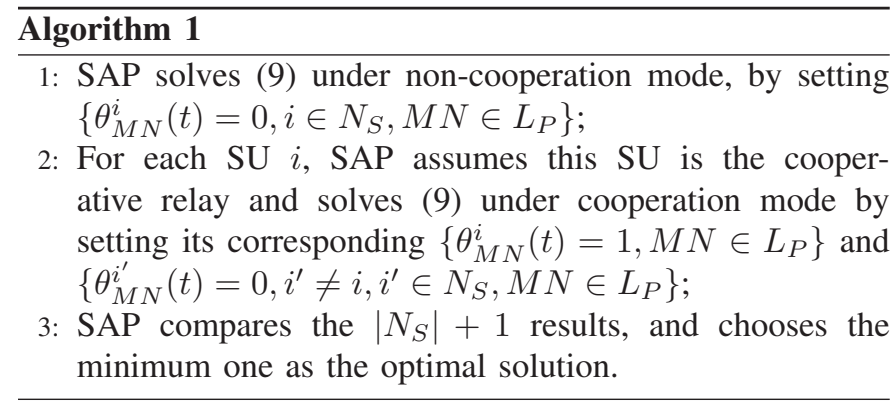

We can prove that through running the admitted control and network control algorithms above for a period of time, the long-term optimum of (8) can be achieved. Due to the space limitation, we omit the proof here and it can be found in [17].

\section{Performance Evaluation}

We generate a random primary network with 25 primary links within an area of $100 \mathrm{~m} \times 100 \mathrm{~m}$. In each interval, we randomly choose one primary link, and activate it with probability $P r_{b u s y}$. In the same area, there is a secondary network with randomly located SUs and the SAP. Channel gain $h$ is modeled with large-scale path loss and small-scale fading, where large-scale path is composed by path loss and shadow fading. We set $A_{\max }=20, P=P^{\prime}=10, V=70$ and iteration time $t_{\max }=4000$. For each SU, we assume the traffic arrival rate at transport layer follows a Poisson process with mean rate $\bar{\lambda}_{i}$, where $\bar{\lambda}_{i}$ is randomly chosen from $[1,5]$. Moreover, $N_{0}=3.38 \times 10^{-15}$ and $E_{\text {ave }}^{i}$ is randomly selected from $[1,5]$ for each SU $i$.
Under this topology, we compare the throughput performance of the proposed spectrum utilization maximization cooperation (SUM-Cooperation) scheme with the conventional simple cooperation scheme (Sip-Cooperation) [8], [9] and Non-Cooperation schemes [1]. Results are shown in Fig. 4Fig. 6. Here the Sip-Cooperation scheme allocates the secondary transmission period only to the relay SU, and the NonCooperation scheme does not consider cooperative communications between primary and secondary networks.

In Fig. 4, we set $P r_{\text {busy }}=0.3$ and the number of SUs is 5. We plot the long-term secondary network throughput w.r.t. the Poisson mean rate $a \bar{\lambda}_{i}$, where $a$ increases from 1 to 6 . It can be seen that under SUM-Cooperation and Sip-Cooperation schemes, the secondary network throughput increases with $a$; while under the Non-Cooperation scheme, the secondary network throughput becomes steady when $a$ is greater than 3 . This is because when Poisson mean rate $a \bar{\lambda}_{i}$ increases, more secondary traffic could be admitted to the secondary network through SUM-Cooperation and Sip-Cooperation schemes, and thus, the throughput increases dramatically. However, since cooperative gain is not involved in Non-Cooperation scheme, when $a$ becomes large, the secondary network under NonCooperation scheme is not able to admit more traffic and the throughput becomes saturated.

In Fig. 5, we evaluate the network throughput under three schemes with different number of SUs by setting $P r_{\text {busy }}=0.3$ and $a \bar{\lambda}_{i}=3 \bar{\lambda}_{i}$. The result shows that the secondary network throughput under SUM-Cooperation and Sip-Cooperation schemes increases with the number of SUs. The reason is with the cooperative gain in the two schemes, the increasing number of SUs provides more cooperation opportunities in CCRNs, and thus, increases the secondary network throughput. In Non-Cooperation strategy, when the number of SUs is small, the throughput increases with the number of SUs. However, when the number of SUs is larger than 4 , the throughput keeps steady. The initial increase of Non-Cooperation curve may be caused by the increment of total traffic arrival rates when the number of SUs grows from 3 to 4 .

Moreover, we exam throughput performance versus the busy probability of PUs $P r_{b u s y}$, where $a \bar{\lambda}_{i}=3 \bar{\lambda}_{i}$ and the number of SUs is 5. Fig. 6 demonstrates that as Prbusy increases, the opportunities for secondary transmissions decreases, which results in the decrease of secondary network throughput under the Non-Cooperation scheme. However, in the SipCooperation and the proposed SUM-Cooperation schemes, the throughput decreases slowly since cooperation could create transmission opportunities for SUs when PUs are busy.

Finally, it can be seen from Fig. 4-Fig. 6 that the proposed SUM-Cooperation scheme always has advantages over the SipCooperation and Non-Cooperation schemes in terms of secondary network throughput. As a result, the proposed scheme could better utilize the spectrum resource for secondary networks to obtain higher secondary network throughput. 


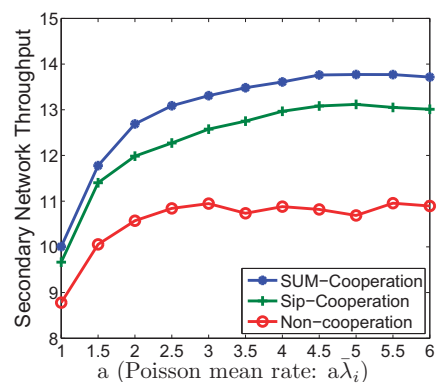

Fig. 4. Secondary network throughput vs. Poisson rate.

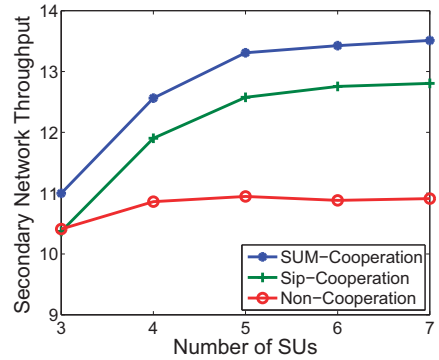

Fig. 5. Secondary network throughput vs. number of SUs.

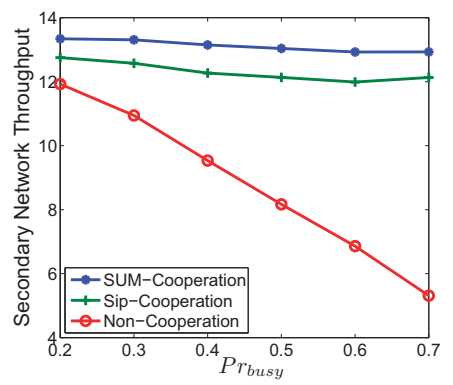

Fig. 6. Secondary network throughput vs. busy probability of PUs

\section{CONCLUSION}

In this paper, to maximize the spectrum utilization among all SUs, we have proposed a resource allocation scheme under dynamic CCRNs with energy constraint. We consider the relay selection and secondary transmission scheduling jointly, and formulate the resource allocation problem from longterm perspective. To obtain the long-term optimum, we design an optimal online algorithm based on instantaneous network setting. Through simulations, we show the proposed scheme could achieve higher secondary network throughput than the existing schemes and provide better spectrum utilization.

\section{ACKNOWLEDGEMENT}

This work was partially supported by the NSFC under grant 91338115 and 61231008, National S\&T Major Project under grant 2011ZX03005-004, 2011ZX03004-003, 2013ZX03004007-003 and 2011ZX03005-003, Shaanxi 13115 Project under grant 2010ZDKG-26, National Basic Research Program of China under grant 2009CB320404, Program for
Changiiang Scholars and Innovative Research Team in University under grant IRT0852, 111 Project under grant B08038, and State Key Laboratory Foundation under grant ISN1002005 and ISN090305. The work of $\mathrm{H}$. Yue and Y. Fang was partially supported by the U.S. National Science Foundation under grant CNS-1343356. The work of M. Pan was partially supported by the U.S. National Natural Science Foundation under grant CNS-1343361.

\section{REFERENCES}

[1] Y. Hou, Y. Shi, and H. Sherali, "Spectrum sharing for multi-hop networking with cognitive radios," Selected Areas in Communications, IEEE Journal on, vol. 26, no. 1, pp. 146-155, Jan. 2008.

[2] H. Yue, M. Pan, Y. Fang, and S. Glisic, "Spectrum and energy efficient relay station placement in cognitive radio networks," Selected Areas in Communications, IEEE Journal on, vol. 31, no. 5, pp. 883-893, 2013.

[3] A. Goldsmith, S. Jafar, I. Maric, and S. Srinivasa, "Breaking spectrum gridlock with cognitive radios: An information theoretic perspective," Proceedings of the IEEE, vol. 97, no. 5, pp. 894-914, May 2009.

[4] Y. Yi, J. Zhang, Q. Zhang, and T. Jiang, "Spectrum leasing to multiple cooperating secondary cellular networks," in Proc. IEEE ICC, Houston, TX, Dec. 2011.

[5] M. Pan, H. Yue, Y. Fang, and H. Li, "The x loss: Band-mix selection with uncertain supply for opportunistic spectrum accessing," in Proc. IEEE GLOBECOM, Miami,Fl, Dec. 2010.

[6] Y. Xing, C. N. Mathur, M. Haleem, R. Chandramouli, and K. Subbalakshmi, "Dynamic spectrum access with qos and interference temperature constraints," Mobile Computing, IEEE Transactions on, vol. 6, no. 4, pp. 423-433, Apr. 2007.

[7] D. Yang, X. Fang, and G. Xue, "Hera: An optimal relay assignment scheme for cooperative networks," Selected Areas in Communications, IEEE Journal on, vol. 30, no. 2, pp. 245-253, Feb. 2012.

[8] O. Simeone, I. Stanojev, S. Savazzi, Y. Bar-Ness, U. Spagnolini, and R. Pickholtz, "Spectrum leasing to cooperating secondary ad hoc networks," Selected Areas in Communications, IEEE Journal on, vol. 26, no. 1, pp. 203-213, Jan. 2008.

[9] J. Zhang and Q. Zhang, "Stackelberg game for utility-based cooperative cognitiveradio networks," in Proc. ACM MobiHoc, New Orleans, LA, May 2009.

[10] H. Xu and B. Li, "Resource allocation with flexible channel cooperation in cognitive radio networks," Mobile Computing, IEEE Transactions on, vol. 12, no. 5, pp. 957-970, May 2013.

[11] K. Khalil, M. Karaca, O. Ercetin, and E. Ekici, "Optimal scheduling in cooperate-to-join cognitive radio networks," in Proc. IEEE INFOCOM, Shanghai, China, Apr. 2011.

[12] M. Pan, P. Li, Y. Song, Y. Fang, and P. Lin, "Spectrum clouds: A session based spectrum trading system for multi-hop cognitive radio networks," in Proc. IEEE INFOCOM, Orlando, FL, Mar. 2012.

[13] R. Urgaonkar and M. Neely, "Opportunistic cooperation in cognitive femtocell networks," Selected Areas in Communications, IEEE Journal on, vol. 30, no. 3, pp. 607-616, Apr. 2012.

[14] Y. Zhang, N. Meratnia, and P. Havinga, "Outlier detection techniques for wireless sensor networks: A survey," Communications Surveys Tutorials, IEEE, vol. 12, no. 2, pp. 159-170, Second 2010.

[15] M. Vutukuru, H. Balakrishnan, and K. Jamieson, "Cross-layer wireless bit rate adaptation," in Proc. ACM SIGCOMM, New York, NY, Aug. 2009.

[16] H. Ju, B. Liang, J. Li, and X. Yang, "Dynamic power allocation for throughput utility maximization in interference-limited networks," Wireless Communications Letters, IEEE, vol. 2, no. 1, pp. 22-25, Feb. 2013.

[17] M. J. Neely, Stochastic Network Optimization with Application to Communication and Queueing Systems. Morgan and Claypool Publishers, 2010.

[18] M. Neely, "Energy optimal control for time-varying wireless networks," Information Theory, IEEE Transactions on, vol. 52, no. 7, pp. 29152934, Jul. 2006.

[19] M. Lotfinezhad, B. Liang, and E. Sousa, "Optimal control of constrained cognitive radio networks with dynamic population size," in Proc. IEEE INFOCOM, San Diego, CA, Mar. 2010. 